\title{
Die ehemalige St. Georgs-Kapelle zu Rüblingen
}

\section{Geschichte einer verschwundenen Dorfkapelle}

\author{
von Andreas VolK
}

In dem kleinen Weiler Rüblingen, einem Ortsteil der Gemeinde Kupferzell, steht ein Glockenturm mit zwei Glocken und einer Uhr. Dem entsprechend trägt die Dorfstraße den Namen „Am Glockenturm“. An der Stelle des heutigen Glockenturms stand einmal eine kleine Kapelle, welche dem heiligen Georg geweiht war. Das Gotteshaus ist längst verschwunden. Nur einige Hinweise in der heimatgeschichtlichen Literatur erinnern an die Kapelle. Teils weichen die dortigen Angaben von einander ab. Manchmal erweisen sie sich bei näherer Betrachtung auch als falsch. In den Archiven befinden sich viele Unterlagen zu der Kapelle, welche in der Literatur noch gar nicht beachtet wurden, und die - obwohl die Kapelle nicht mehr existiert - interessant genug sind, um sich näher mit der ehemaligen Kapelle zu beschäftigen.

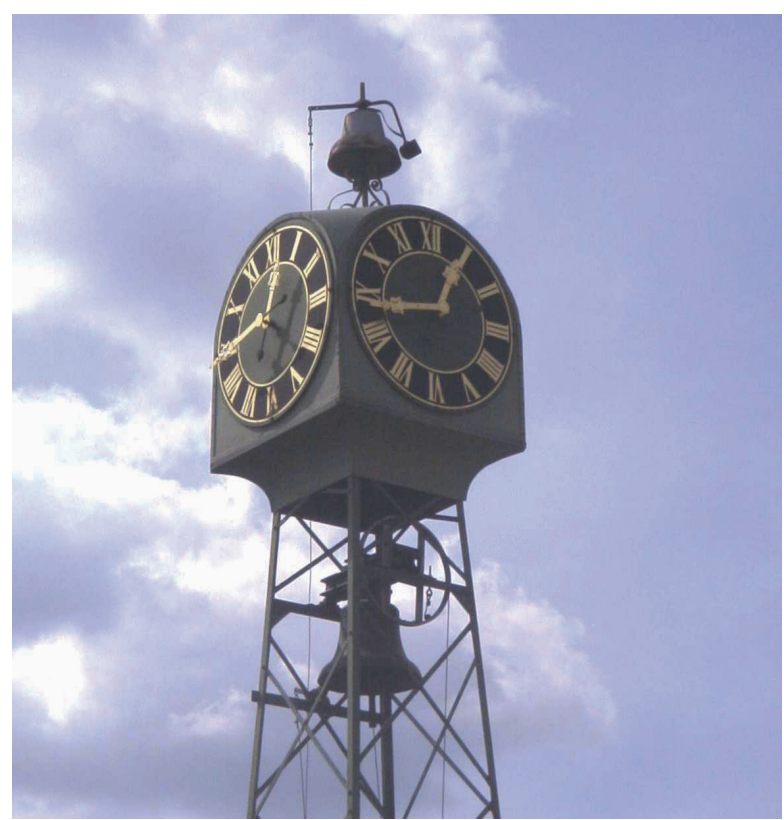

Abb. 1: Der Glockenturm von Rüblingen (Foto: Andreas Volk) 


\section{Die frühesten Nachweise}

Wie bei vielen Kirchen sagt man auch der St. Georgs-Kapelle in Rüblingen nach, dass sie eine der ältesten des Bezirkes, sogar in ganz Franken gewesen sein soll. Die tatsächlichen Ursprünge dieses Gotteshauses liegen im Dunkel. Dass gerade eine der beiden Glocken auf dem heutigen Glockenturm das älteste Zeugnis von der Kapelle ist, ist eine Tatsache, die man zunächst einmal nicht vermuten würde. Während die obere, kleinere Glocke eine Stahlglocke aus der Zeit nach dem Ersten Weltkrieg ist, ist die untere, größere Glocke eine Bronzeglocke aus dem 15. Jahrhundert ${ }^{1}$. Sie trägt eine Schulterinschrift aus gotischen Minuskeln mit folgendem Text: ave maria gracia plena dominus. Die Glocke trägt keine Jahreszahl, auch keinen Namen eines Glockengießers und wurde bisher nur vage in die Zeit um 1500 datiert. Doch durch die Forschungen von Harald Drös ist seit 2008 bekannt, dass die Rüblinger Glocke aus dem ersten Viertel des 15. Jahrhunderts stammt. Wenn man unterstellt, dass diese Glocke schon immer für die Rüblinger Kapelle bestimmt war, so könnte demnach schon im ersten Viertel des 15. Jahrhunderts in Rüblingen eine Kapelle bestanden haben, die mit einer Glocke ausgestattet wurde. Dies passt zu der ersten urkundlichen Nennung, welche für das Jahre 1431 überliefert ist ${ }^{2}$. Laut dieser Urkunde gab Margaretha von Enslingen am 13. Juli 1431 (sant Margarethen Tag) ein Gut für $7 \mathrm{fl} \mathrm{dem} \mathrm{lieben}$ Heren Sant Jörgen zu Ryblingen ${ }^{3}$. Mit diesem „,lieben Herrn Sankt Jörgen“ ist wohl die Rüblinger Kapelle bzw. deren Patron gemeint. Eine weitere Urkunde kennt man aus Wibels „Hohenlohische[r] Kyrchen- und Reformationshistorie“. Im ersten Band seiner 1752 erschienenen Kirchengeschichte berichtet er: Rüblingen, eine Stunde von Kupferzell, hat eine Kyrche, welche ein Filial von Eschenthal ist, A. 1454 verkaufte das Kloster Gnadenthal seine Güter zu Holtzhausen dem Heiligen in Rüblingen, welchen sie hernach Graf Albrecht von Hohenlohe A. 1486 mit Einwilligung der Pfarrers zu Eschenthal gegen andere ausgewechselt ${ }^{4}$. Im dritten Teil seiner Kirchengeschichte, dem "Codex diplomaticus“ von 1754, bringt Wibel einige bruchstückhafte Ergänzungen dazu ${ }^{5}$. Die Autoren

1 Zur Beschreibung dieser Glocke siehe: Evangelisches Pfarrarchiv Eschental sowie Landeskirchliches Archiv Stuttgart A 29 Bd. 1483 und: Harald Drös: Die Inschriften des Hohenlohekreises. Teil 1. Wiesbaden 2008, S. $125 \mathrm{f}$.

2 Der Landkreis Öhringen. Amtliche Kreisbeschreibung. Band II. Öhringen 1968, S. 165; Das Land Baden-Württemberg - Amtliche Beschreibung nach Kreisen und Gemeinden. Bd. IV. Stuttgart 1980, S. 214; Jürgen Hermann Rauser: Kupferzeller Heimatbuch. (= Heimatbücher Hohenlohekreis 17). Ingelfingen 1985, S. 174.

3 HStA Stuttgart B 352 U 96.

4 Johann Christian Wibel: Hohenlohische Kyrchen- und Reformationshistorie, Onolzbach 1752, S. 175 .

5 Johann Christian Wibel: Hohenlohische Kyrchen- und Reformationshistorie. Dritter Theil. Codex Diplomaticus. Onolzbach 1754, S. 64: Ich Contz Strecker und Ich Adam Gundel, der Zeit Heiligenpfleger zu Ryblingen ... der Capellen zu Ryblingen zwey Gütere zu Holtzhausen ... Ich Johannes Zaner (al. Zawer) Pfarrer zu Eschenthal; S. 79: Anno 1454. Wir Swester Barbara Abtissin zu Gnadental ... dem Heiligen zu Ryblingen 
der 1865 erschienenen Oberamtsbeschreibung Öhringen brachten dieses Holtzhausen mit Jungholzhausen in Zusammenhang ${ }^{6}$. Lange Zeit war die Urkunde von 1454 nicht auffindbar. Dank den Verzeichnungsarbeiten im Hohenlohe-Zentralarchiv in Neuenstein ist sie nun wieder zugänglich, so dass Wibels Fragmente ergänzt werden können: Demnach verkauften die Äbtissin Barbara von Stetten und der Konvent des Klosters Gnadental an den Heiligen zu Rüblingen und dessen Pflegern Gülten und Güter in Holzhausen ${ }^{7}$. Auch in dieser Urkunde wird zwar noch nicht explizit eine Kapelle genannt, wohl aber die Heiligenpfleger. Dafür existiert vom Jahre 1454 eine weitere Urkunde, die (außer der Nennung ihrer Jahreszahl ${ }^{8}$ ) noch nicht in der Literatur berücksichtigt wurde. Danach übergab Engelhard Gockenbach Gelder und Güter, nämlich die Stockwießen und den Zehendacker gelegen zu Heßelbrune [Hesselbronn] der Cappellen Sant Jorgn zu Rieblingen und den Heyligenpflegern daselbst ${ }^{9}$. Vom 9. Juli 1461 gibt es noch einen Kaufbrief. Darin verkaufen Hans Schneider von Morspach und Margareth, seine Tochter, der Cappeln Sant Jorgen zu Ryblingen und ire Pflegern an ir stat da selbst mit Namen Petter Hoffmann und Contz Kistner die Hälfte des großen und kleinen Zehnts zu Bulingswyler ${ }^{10}$ um $30 \mathrm{fl} \mathrm{rh}^{11}$. Dazu findet sich eine Ergänzung im Eschentaler Pfarrgültbuch von 1640, nach der der Verkauf der Hälfte des großen und kleinen Zehnts in Lieblisweiler ${ }^{12}$ durchgeführt wurde, damit jährlich Schneiders Freünd und Eltern [...] an der Kirchwey gedacht wer$\mathrm{de}^{13}$.

\section{Ein archivalisch überliefertes Baujahr}

Diesen vier frühen Urkunden und der Glocke widerspricht das bisher bekannte Baujahr der Rüblinger Kapelle - nämlich das Jahr 1468. Im Jahre 1832 wollte man über dem Kirchenschiff der Kapelle eine Schulstube einrichten. Der Öhringer Architekt Reinhardt verfasste hierzu die Baupläne und Kostenvoranschläge und lieferte interessante Beschreibungen der Kapelle: Die Kirche zu Rüblingen ist nach anliegenden Rissen und [recte wohl: um] 1468 erbaut worden. Sie hat ringsum einen steinernen Stock und über der Kirche einen Stock von Holz, 8'

6 OAB Öhringen. Stuttgart 1865, S. 212.

7 HZA Neuenstein La 150 U 56 27: Demnach wir Swester Barbara von Stetten Abbtissinne und der Convent gemeynlich des Closters zu Gnadentale recht und redlichen verkaufft und zu kauffen geben haben den Heiligen zu Ryblingen und ihren Pflegern mit Namen Peter Hoffman und Jorigen Heffnern und allen iren Nachkomen die Gülten und Güter mit iren Zugehorungen gelegen zu Holtzhusen.

8 HZA Neuenstein Wa 82.

9 Der Landkreis Öhringen (wie Anm. 2), S. 165.

10 Bühlingsweiler, abgegangener Weiler bei Rüblingen.

11 HStAS B 352 U 105; datiert auf: Donderstag nehest nach sant Kylians Tag.

12 Leipoldsweiler, Gemeinde Untermünkheim.

13 Evangelisches Pfarrarchiv Eschental. 
hoch und dann das Dachwerk. In einer anderen Textstelle schrieb er: Bei Aufnahme der Kirche hat sich der Unterzeichnete überzeugt, daß der untere steinerne Giebel nur aus lauter kleinen Heuchelsteinen gebaut ist, mit Sicherheit zum Errichten einiger Fenster nicht angegriffen werden kann ${ }^{14}$. Die Zahl 4 der Jahreszahl 1468 hat der Architekt in der am Kirchengebäude angebrachten Schreibweise wiedergegeben, nämlich in Schlingenform (eine halbe 8). Wie passt diese späte Jahreszahl zu den Urkunden und der Kirchenglocke aus der Zeit davor? Des Rätsels Lösung könnte ein Neubau der Kapelle sein. Möglicherweise wurde eine ältere Kapelle wegen Baufälligkeit abgebrochen und wieder aufgebaut. Oder es handelte sich um eine Erweiterung oder einen Anbau an der Kapelle. Wo der Inschriftenstein angebracht war, geht aus der Beschreibung des Architekten nicht hervor.

\section{Weitere urkundliche Nennungen}

Durch die Urkunde aus dem Jahre 1454, welche in Wibels Kirchengeschichte genannt ist, wurde schon auf eine andere Urkunde aufmerksam gemacht, nämlich jene vom 12. Januar Jahre 1486. Es ist dies ein Tauschbrief der Heiligenpfleger zu Rüblingen: Contz Strecker und Adam Gundel derzeit Heyligen Pfleger zu Ryblingen tauschen mit Vergonnen Willen und Wissen des Kraft von Hohenlohe und Ziegenhain ${ }^{15}$ ein Gut zu Steinkirchen das jetzo bauet und besizet Paul Zymmermann gegen ihre der Capellen zu Ryblingen zwey Guter zu Holtzhausen. Der Eschentaler Pfarrer Johannes Zawer [...] Sanct Johans Orden gibt in der Urkunde seine Zustimmung zu dem Güterwechsel ${ }^{16}$.

Die nächste Urkunde zur Rüblinger Kapelle stammt vom 22. Mai 1514: Hainz Scheyfer zu Rieblingen bekennt, daß Hanß Seytzinger und Jörig Strecker der Zeyt bayde Hailigen Pfleger des hailigen Ritters und Märttererß Sant Georigen zu Rieblingen mit Zustimmung des erwürdig und fürnemmen Hanß Dyernen, damals Vogt und Amtmann zu Waldenburg, an ihn um jährlich 8 B d zu Erblehen verliehen haben den Sandt Jörigen Busch und ist ungevüerlich uff zwen Morgen, auch so ist er veraynt unnd versteindt. ${ }^{17}$. Eine weitere Urkunde stammt aus dem Jahre 1520. Es ist dies ein lateinisch abgefasster Ablassbrief des Würzburger Weihbischofs Johannes Pettendorf (Weihbischof von 1512 bis 1525), dem Generalvikar des Würzburger Bischofs Konrad von Thüngen (Bischof von 1519 bis 1540). Es wurde Ablass gewährt für alle, welche an gewissen Tagen in der $\mathrm{Ca}$ pella in Riblingen an den Altären der Schutzpatrone Buße tun und zum Heiligenvermögen eine Stiftung machen ${ }^{18}$. In dem Ablassbrief werden zehn Altäre aufge-

14 Gemeindearchiv Kupferzell, Bestand Feßbach, A 335.

15 HZA Neuenstein La 150 U 56 32/4, datiert auf Donerstag nach der heyligen dreyer König Tag.

16 Jungholzhausen, Gemeinde Braunsbach, oder abgegangener Ort bei Steinkirchen.

17 HStA Stuttgart B 352 U 128, datiert auf Montag in der Creutzwochen.

18 HStA Stuttgart B 352 U 131. 
zählt. Es waren dies die Altäre des Georg, Cyriak, Laurentius, Saturnius, Margaretha und die 11.000 Jungfrauen. Diese Altäre standen im Chor. Im Südteil der Kapelle nennt die Urkunde die Altäre von Maria, Anna, Ulrich und Michael. Am interessantesten an dem Ablassbrief ist die Erwähnung, dass die Ablassempfänger beim Schlag der Ave-Maria-Glocke (pulsum salutationis angelice) drei Ave Maria sprechen sollen. Hier haben wir also eine urkundliche Erwähnung der anfangs erwähnten Glocke.

\section{Die Heiligenpflege}

Aus dem 15. und 16. Jahrhundert liegen sieben Urkunden vor, welche eine Kapelle in Rüblingen und eine Heiligenpflege bezeugen. Die Heiligenpfleger führten über das Kirchenvermögen, welches sie verwalteten, eine Heiligenrechnung. Deren älteste stammen aus Zeit von 1543 bis 1553, die als Hailligenpfleger Sanct Jorgen Kirch zue Ryblingen den Johann Seitzinger und Johann Ilzhöfer nennen ${ }^{19}$. Die Einnahmeseite bewegte sich jährlich um 50 fl. Über die Einnahmen von 1557 berichtet auch ein Gültbuch der Georgs-Kirche ${ }^{20}$. Von 1583 bis 1614 wurde die Heiligenrechnung zusammen mit der von Westernach geführt $^{21}$. Als Rechner fungierte 1583/84 der Bürger Hans Beingesser aus Waldenburg, von 1586 bis 1614 der Wirt Johann Sebastian Wörner aus Westernach $(†$ 15. März 162622). Von 1614 bis 1622 wurde zusammen mit Eschental eine Heiligenrechnung geführt ${ }^{23}$. Rechner war immer noch der Westernacher Wirt Johann Sebastian Wörner. Dann kam der Dreißigjährige Krieg, unter dem Rüblingen schwer zu leiden hatte. In einem 1640 angelegten Verzeichniß waß vor Güether und Häußer in dem Ampt Zell oedt und wüest ligen heißt es, dass man in Rüblingen 15 öde Güter zählte ${ }^{24}$. Es war also mehr als die Hälfte von Rüblingen zerstört oder verlassen. Noch 1681 wird im neu angelegten Rüblinger Lagerbuch von manchem Hofgut berichtet, das im Dreißigjährigen Krieg eingefallen und noch nicht wieder aufgebaut worden ist ${ }^{25}$. 1631 scheint die letzte Heiligenrechnung ordnungsgemäß geführt worden zu sein. 1651 wurde auf dem Waldenburger Amtshaus eine Abrechnung über den Eschentaler und Rüblinger Heiligen vorgenommen $^{26}$. Im Jahre 1652 wurde ein Heiligenzinsbüchlein über die renovierten Eschentaler und Rüblinger Heiligenkapitalien neu angelegt ${ }^{27}$. Ab 1652

19 HZA Neuenstein Wa 265 Bü 1949 bis 1951.

20 HZA Neuenstein Wa 260 Bd. 185; zur Datierung enthält das Gültbuch den Eintrag: Sant Jorgn

Kirch zu Ryblingen erneuert uff Sambstag nach Paulus Bekehrung (28. Januar) 1557.

21 HZA Neuenstein, Wa 265, Bü 1952 bis 1981.

22 Landekirchliches Archiv Stuttgart, KB 748.

23 HZA Neuenstein Wa 265 Bü 1815 bis 1817.

24 HZA Neuenstein Wa 140 Bü 152.

25 HZA Neuenstein Wa 260 Bd 84.

26 HZA Neuenstein Wa 265 Bü 1819.

27 Gemeindearchiv Kupferzell, Bestand Eschental, A 196. 
wurde dann nur noch eine Heiligenrechnung in Eschental geführt, worin ist es heißt: Anno 1634 bis anno 1652 nichts eingenommen oder ausgegeben worden, wird also diesmals damit wieder ein Anfang gemacht ${ }^{28}$. Von da ab unterhielt die Eschentaler Heiligenpflege aus den Stiftungszinsen auch die Kapelle in Rüblingen. Da die Heiligenpflegen von Eschental und Rüblingen zusammengelegt waren, war von jedem Ort ein Heiligenpfleger tätig. Ab 1652 war dies für Rüblingen Hans Strecker der Ältere.

\section{Auszüge aus den älteren Heiligenrechnungen}

Durch die Heiligenrechnungen sind wir über die baulichen Unterhaltungen der Kapelle in Rüblingen unterrichtet, wobei in den ältesten Heiligenrechnungen von 1546 bis 1548 nur wenige Bauausgaben vorkommen, die den Zaun um den Kirchhof und die Reparatur der Kirchtüre betreffen ${ }^{29}$. Eine Uhr wurde 1606 von der Rüblinger Gemeinde angeschafft: Wie wir ein Uhrwercklin erkauft und selbiges in die Kirchen ordern lassen, damit in unsern Geschäfte nach der Zeit uns hätten zu richten ${ }^{30}$. Gleichzeitig wurde die Stelle eines Mesners geschaffen: Michel Seizinger hierzu sich will gebrauchen lassen, welcher auch mit der Uhren nach Notturft und Gebür weiß und kann umbgehen. Dafür erhielt er Fron- und Dienstbefreiung.

1620 wurden $2 \mathrm{fl}$ für etlich Stüehl in die Rüblinger Kirchen aufgewendet ${ }^{31}$. Im Jahre 1622 wurden $11 \mathrm{fl} 10 \mathrm{~b} 6 \mathrm{x}$ ausgegeben von der Kirchen zu Rübling zu deckhen und zu übersteigen wie auch den Thurm von neuern uff zu brechen und zu ligen ${ }^{32}$. Ab 1652 unterhielt die Eschentaler Heiligenpflege aus ihren Stiftungszinsen die Kapelle in Rüblingen. So enthält die Eschentaler Heiligenrechnung von 1652 folgenden Eintrag: 12 x dem Mäurer zue Rüblingen durch Han $\beta$ Streckhern alda bezahlt für seinen Bestandt am Rüblinger Kirchlein ${ }^{33}$ oder $1 \mathrm{fl}$ 20 x dem Ziegler zue Kupferzell für 8 Centner Kalch zue der Kirchhofmauer zue Rüblingen.

Auch die Ausgaben für die Inneneinrichtung der Rüblinger Kapelle wurden aus den Eschentaler Stiftungszinsen bestritten. So heißt es in der Heiligenrechnung von 1653/55: 6 fl umb ein lederne Canzelwerkh mit silbernen Schnüren dem Deppichmacher zue Ohnolzbach in Rüblinger Kirch ${ }^{34}$. In der Eschentaler Heiligenrechnung von 1655/56 findet sich folgender Eintrag: 50 x zalt Heyligenpfle- 
ger Hanß Streckher umb 2 Scheiben zuer Uhr in die Rüblinger Kirch ${ }^{35}$. 1658/59 wird ein Langhaus erwähnt. Dem Rüblinger Mauer Georg Ufinger wurde Geld ausgezahlt, um im Langhauß Ziegel einzustoßen ${ }^{36}$. Auch wurden $3 \mathrm{fl} 41 \mathrm{x}$ dem Ziegler zue Kupfferzell für 24 Centner Kalch und $5 x$ für Ziegel zur Rüblinger Kirchmauer und $3 \mathrm{fl}$ dem Mäurer Georg Ufinger von solcher Mauer wurden ufzuführen gegeben ${ }^{37}$. Laut der Heiligenrechnung von 1660/1661 wurden $1 \mathrm{fl} 4$ $\mathrm{x}$ der Gemeind zue Rüblingen zu einem Tächlein uff die Kirchenstaffel und Raparirung de $\beta$ Grettelhaußes erstattet ${ }^{38}$. Über Kirchenstühle berichtet die Eschentaler Heiligenrechnung von 1698/99. Da wurde dem Georg Simon Bentz zu Rüblingen vor underschiedliche Flikarbeit an denen Kirchenstühlen in der Rüblinger Kirchen Geld ausbezahlt ${ }^{39}$. Leider ist über die Anzahl und Umfang des Kirchengestühls nichts bekannt.

\section{Die Verhältnisse 1681 und 1682 und das Kirchenbauwesen im 18. Jahrhundert}

Im Jahre 1681 wurde das Rüblinger Gült- und Lagerbuch angelegt, in dem die kirchlichen Verhältnisse in Rüblingen ausführlich beschrieben sind. Ein Moderationsprotokoll, das 1682 anlässlich der hohenlohischen Landesteilung angelegt wurde, ergänzt die Informationen von 1681 insbesondere im Hinblick auf den baulichen Zustand der Kapelle ${ }^{40}$. Besonders hervorzuheben ist, dass nach alter örtlicher Tradition die Rüblinger Kapelle ursprünglich die Mutterkirche von Eschental gewesen sein soll, während im späten 17. Jahrhundert die Verhältnisse genau umgekehrt waren: Rüblingen war jetzt Filial von Eschental.

Der Eschentaler Heilige unterhielt weiterhin die Kapelle in Rüblingen. So wurden 170116 fl 30 x an Niclas Strobeln, Maurern von Tyrol, vor Reparirung der allenthalben sehr bawfälligen Kirchen zue Rüblingen gegeben ${ }^{41}$. Auch laut der Heiligenrechnung von 1710/13 war wieder ein aus den Alpen stammender Handwerker tätig: Maurer Egold außm Tyrol erhielt $1 \mathrm{fl} 30$ x, weil er daß Dach uff der Rüblinger Kirche umbgedecket und den Knopff hinauff gemacht ${ }^{42}$. Und im Juli 1711 wurden dem Maurer Deschler von Döttingen 21 fl wegen der Rüblinger Kirchen und Kirchhoffsmauer per Accord gegeben ${ }^{43}$. 1731/32 erhielt der namentlich nicht genannte Maurer zu Döttingen 1 fl 38 x für Reparaturen an der

Ebd., R 271.

Ebd., R 274.

Ebd.

Ebd., R 276.

Ebd., R 277.

Vgl. dazu Anhang 1 und 2.

HZA Neuenstein Wa 265 Bü 1848.

Gemeindearchiv Kupferzell, Bestand Eschental, R 284.

Ebd., R 295. 
Kirche zu Rüblingen ${ }^{44}$. Solche kleineren Arbeiten erscheinen auch in der Eschentaler Heiligenrechnung von 1739/40: Der Schultheiß von Rüblingen erhielt für allerhand Baumaterialien zur Rüblinger Kirche 3 fl $21 \mathrm{x}^{45}$. Eine ganze Anzahl verschiedener Handwerker nennt die Heiligenrechnung von 1740/41. Der Nagelschmidt Faad in Kupferzell erhielt vor Nägel in die Rüblinger Kirchen $1 \mathrm{fl} 13$ x. Deutlich höher war der Lohn für den M[eister] Zimmermann Curr zu Kupferzell, der 17 fl erhielt, während der M[eister] Maurer Johann Michael Kümmerer zu Kupferzell 9 fl 52 x, der M[eister] Glaßer zu Cünzelsau 10 fl 30 x bekam. Dessen Jung erhielt $4 \mathrm{fl}$ als Trankgeld, 5 fl der Schmied Schmuderer zu Rüblingen ${ }^{46}$. Insgesamt beliefen sich also die Reparaturkosten 1740/41 auf 47 fl 35 x.

Während 1745/46 nur $18 \mathrm{fl} 4 \mathrm{x}$ an den Maurermeister Johann Michael Kümmerer in Kupferzell vor die Rüblinger Kirchhofmauer bezahlt wurden ${ }^{47}$, stand 1759/60 wieder eine etwas größere Renovierung an, die - genau wie 1740/41 an verschiedene Handwerker ging: $21 \mathrm{fl} 30 \mathrm{x}$ an Meister Kümmerer, Maurer in Kupferzell, 5 fl 56 x an Herrn Haagen als dem Oberwirth in Kupferzell vor Kalch und Ziegel, $3 \mathrm{fl}$ an Meister Ziegler in Steinkirchen vor Kalch, $1 \mathrm{fl} 20 \mathrm{x}$ an Meister Schmuderer, Schmidt zu Rüblingen. 30 x erhielt Johann Georg Gundel in Rüblingen vor einen grosen Sandstein zu der dasigen Kirchenthür, 2 fl erstaunlicherweise der Maurersgesell Fünffer, vor die Rüblinger Uhrtafel zu repariren und mahlen, er hatte also eher eine Malerarbeit ausgeführt ${ }^{48}$. Insgesamt kamen bei den Reparaturen von 1759/60 demnach 34 fl 16 x zusammen.

\section{3 wird eine neue Kirchenglocke gegossen}

Wesentlich teurer als die bisher genannten, insgesamt doch bescheidenen Reparaturen war das Gießen einer neuen Glocke 1732/33. In der Eschentaler Heiligenrechnung heißt es dazu: Von der einen Glocke zu Rüblingen, die versprungen gewesen und umgegossen worden, wurde bezahlt an den Rothgießer Röschen zu Morsbach $54 \mathrm{fl} 15$ x ${ }^{49}$. Auch wenn hier der Name des GlockengieBers mit Rösch angegeben ist, so ist doch klar, dass damit der Morsbacher Glockengießer Johann Leonhard Lösch $\left(\dagger 4\right.$. September $\left.1765^{50}\right)$ gemeint ist. Über den Glockenguss Löschs sind in den Rechnungen der Eschentaler Heiligenpflege einige Akten erhalten. Darin wird genau aufgeschlüsselt, wie die 54 fl $15 \mathrm{x}$ zustande gekommen waren. Der entsprechende Rechnungsbeleg vom 27. No-

44 Ebd., R 288.

45 Ebd., R 295.

46 Ebd., R 296.

47 Ebd., R 301.

48 Ebd., R 315.

49 Ebd., R 289.

50 Deutscher Glockenatlas. Württemberg und Hohenzollern. Deutscher Kunstverlag, München 1959, S. 100. 
vember 1733 ist wegen seiner Details zum technischen und organisatorischen Ablauf eines Glockengusses sowie zu den verwendeten Materialien so bemerkenswert, dass er als Anhang im Gesamtzusammenhang aufgeführt ist ${ }^{51}$. Nicht nur der Glockengießer war an der Arbeit beteiligt, sondern auch ein Schreiner, der die Aufhängevorrichtungen auf dem Kirchturm herzustellen hatte. Die fürstliche Herrschaft Hohenlohe-Langenburg hatte $7 \mathrm{fl} 30 \mathrm{x}$ zu der neu gegossenen Glocke beigesteuert. Dass diese Unterstützung von Hohenlohe-Langenburg erfolgte und nicht von Hohenlohe-Schillingsfürst, rührt daher, dass Eschental, obwohl Kupferzeller Amtsort, zu dieser Zeit gegen Wiederkauf an Hohenlohe-Langenburg abgetreten war.

Nicht minder aufschlussreich sind die in den Rechnungsbeilagen erhaltenen Absprachen, die der Eschentaler Pfarrer Georg Friedrich Gronbach mit dem Glockengießer Lösch traf. Zunächst findet sich ein Bericht vom 4. Oktober 1733 wegen Umgießung der zersprungen Rüblinger Glocken von Pfarrer Gronbach an die Standesherrschaft, in der er über die Art und Weise der Herstellung der Glocke einen Beschluss erbittet. Erneut werden wieder Einzelheiten über die Glockengussarbeiten und die verwendeten Materialien genannt ${ }^{52}$. Das Antwortschreiben der Standesherrschaft kennen wir nicht, doch wurden bereits am 8 . Oktober 1733 weitere Modalitäten mit dem Glockengießer Lösch vereinbart ${ }^{53}$. Insbesondere sollte die neue Glocke dasselbe Gewicht wie die alte haben, wofür Lösch - wie auch für die Qualität der neuen Glocke - garantieren musste. Es haben sich in den Rechnungsbeilagen sogar die Waagzettel der alten und neuen Glocke erhalten. Man ersieht daraus, dass auf eine genaue Einhaltung der Absprachen geachtet wurde ${ }^{54}$.

Aus den genannten Akten erfährt man also, dass eine alte, zersprungene Glocke neu umgegossen wurde. Von wann die alte Glocke und wer deren Hersteller war, geht aus den Akten nicht hervor. Ebenso wenig geht hervor, dass eine Absprache zu einer Glockeninschrift getroffen wurde. Aber dass es eine solche Inschrift gegeben hat, verrät ein Blatt in den Unterlagen des Evangelischen Pfarramtes Eschental. Danach soll die Glocke folgende Inschrift gehabt haben: Das man umgossen hat mich von neuem läuten hören kann hat Graf Ludwig Gemeind und Heilig Entgeld gethan im 1733 Jahr als Georg Friedrich Gronbach Pfarrer war. Gegossen in Morschbach von Friedrich Lösch ${ }^{55}$. Hier fällt sofort der Name Friedrich Lösch ins Auge. Aus den Akten der Heiligenpflege ist bekannt, dass der Glockengießer Johann Leonhard Lösch hieß. Woher diese Abweichung kommt, ob es sich um einen Schreibfehler in dem Schriftstück handelt, lässt sich nicht mehr feststellen, da die Glocke nicht mehr vorhanden ist. 


\section{Der Verfall der Kapelle und ihr Zustand Ende des 18./Anfang des 19. Jahrhunderts}

Obwohl viele Reparaturen und Ausbesserungen vorgenommen wurden, konnte der Verfall der Kapelle in Rüblingen nicht aufgehalten werden. Aus einem Schreiben des Amts Kupferzell vom 26. Oktober 1787 geht hervor, daß die dortige Kirche sich so baufällig befinde, daß [...] sie gezwungen wären nach Eschental in die Kirche zu gehen, welches viele alte Leute ihres Orts dem Gottesdienst beizuwohnen hindere. Darauf erging der herrschaftliche Befehl, dass die Mängel abgestellt werden, damit man wieder ohne Lebensgefahr in die Kirche gehen könne. Der bereits nach Rüblingen entsandte Hofzimmermann von Kupferzell sollte von dem Collectengeld bezahlt werden ${ }^{56}$. Schon 1786 war die Genehmigung erteilt worden, zur Reparatur des Kirchleins in Rüblingen Kirchenkollekten durchzuführen ${ }^{57}$.

Wie viel Ende der 1780er Jahre renoviert worden war, ist nicht ersichtlich. Um 1820 jedenfalls war der Zustand der Kapelle nicht gut. Im Messprotokoll von 1817 wird das Gotteshaus im Weiler genannt, welches der Commun gehörte ${ }^{58}$. Auch nach dem Brandschadensversicherungskataster von 1821 gehörten Kirche samt Thurm der Gemeinde ${ }^{59}$.

Anfang des 19. Jahrhunderts gab es Streitigkeiten über die Unterhaltungskosten an der Rüblinger Kapelle. Die Pfarrgemeinde Eschental war nicht gewillt, die Kosten an der Kapelle zu übernehmen. Es entstanden dabei auch Diskussionen darüber, ob in Rüblingen überhaupt noch eine Kapelle notwendig sei. Zahlreiche Streit- und Prozessakten sind darüber vorhanden. Diese Akten geben einen interessanten Einblick in Geschichte und Tradition zur Rüblinger Kapelle. Die Angaben in den Akten sollten aber mit Vorsicht benützt werden. So heißt es zum Beispiel in einem Schreiben vom 28. Mai 1819: Das Alter ihrer [der Rüblinger] Kirche, welche nach einer am Hauptgebäude angebrachten Jahreszahl anno 1068 erbaut worden und folglich 751 Jahr alt seye ${ }^{60}$. Es ist anzunehmen, dass dieses Baujahr nicht richtig wiedergegeben ist, sei es, weil die angebrachte Jahreszahl schlecht zu erkennen war oder nicht richtig gedeutet wurde. Auch sagten die älteren Bürger von Feßbach und Kuhbach - welche beyde Orte eine starke halbe Stunde von Rüblingen entfernt sind - etwas Ähnliches aus, was schon 1681/82 dokumentiert worden war, nämlich dass sie es nicht anders wissen und von unsern Eltern und Voreltern nie anders gehört haben, daß in ganz alten Zeiten die Gemeinden zu Feßbach und Kuhbach - ehe sie nach Eschenthal und weiter nach Kupferzell getheilt wurden - Filiale von der Kirche zu Rüblingen

HZA Neuenstein Wa 140 Bü 41.

HZA Neuenstein Sf 10 Bü 546.

HZA Neuenstein Wa 260 B 210.

Gemeindearchiv Kupferzell, Bestand Feßbach, B 140.

Gemeindearchiv Kupferzell, Bestand Feßbach, A 334. 
gewesen seyen. Außerdem befinde sich bis in die Gegenwart - also 1819 - auf der Kuhbacher Markung auch ein alter auf Rüblingen führender Weeg [...], welchen man bis daher den Rüblinger Kirchweeg nennt und wohl von nichts anderem als dem Kirchengang dahin seinen Namen erhalten haben mag. Im darauf folgenden Rechtsstreit wurde im Jahre 1821 der Heiligenpflege Eschental die Baupflicht an der Rüblinger Kapelle ausdrücklich auferlegt. Durch Entscheidung der königlichen Regierung in Ellwangen wurde die Heiligenpflege Eschental und aushilfsweise die ganze Kirchengemeinde Eschental zur vorläufigen Tragung der Baukosten der Rüblinger Kapelle für schuldig erkannt ${ }^{61}$. Als Begründung nannte die Jagstkreisregierung die im Lager- und Gültbuch von 1681 erwähnte Baupflicht des Heiligen von Eschental. Auch aus der Heiligenrechnung von 1700/1701 gehe ebenfalls hervor, dass die Heiligenpflege von Eschental Gelder für die Reparierung der baufälligen Rüblinger Kapelle aufgewendet hatte.

Nachdem die Baupflicht an der Kapelle in Rüblingen geklärt war, fand folgende Beschreibung in die Eschentaler Pfarrbeschreibung von 1828 Eingang: Ebenso steht auch das Eigentum der Filialkirche zu Rüblingen, wohin eine Stunde von hier aus ist, dem Heiligen [von Eschental] zu. Diese Kirche ist klein, äußerst niedrig, innerhalb schwer darin zu predigen ist. Sie hat keine Sakristei, aber einen Turm, zwei Glocken und eine Uhr62.

Wie schon zu Anfang erwähnt, wollte man im Jahre 1832 über dem Schiff der Rüblinger Kapelle eine Schulstube einrichten ${ }^{63}$. Doch die Planungen wurden nicht umgesetzt. Stattdessen dachte man 1837 darüber nach, Rüblingen nach Döttingen einzupfarren. Aber wegen der Engräumigkeit von Kirche und Schule in Döttingen blieb dieses Gesuch aus Rüblingen unbeachtet ${ }^{64}$.

\section{Dem Abbruch entgegen}

Auch die im 19. Jahrhundert vorgenommenen Reparaturen konnten den Verfall der Kapelle nicht aufhalten Schließlich wurde die Kapelle in Rüblingen zum 1. Mai 1863 wegen Baufälligkeit geschlossen. Der Eschentaler Pfarrbericht von 1864 beschreibt den schlechten Zustand der Kapelle: Die Kirche, ohne Orgel und Sakristei, ja ohne einen Stuhl für den Geistlichen, ist im Laufe des letzten Jahres durch Anordnung des Königlichen Oberamts Öhringen geschlossen worden, weil sich im Chor des Turms in dem Gewölbe und an den Seitenmauern gefährlich erscheinende Risse zeigten. Da aber auch nach den vom Bezirksbauamt getroffenen Schutzmaßnahmen eine weitere Senkung der Mauern sich er-

61 Evangelisches Pfarrarchiv Eschental.

62 Evangelisches Pfarrarchiv Eschental.

63 Gemeindearchiv Kupferzell, Bestand Feßbach, A 335.

64 HZA Neuenstein Ki 10 Nr. 19/Lit. A/273. 
gab, so ist seit 1. Mai die Kirche nicht mehr dem Gottesdienst geöffnet. Die Erbauung einer neuen Kirche ist absolut geboten, da alle Reparaturen vergeblich wären, wenn nicht etwa ein Betsaal an ihre Stell tritt oder dieselbe ganz eingeht. Darüber, wer die Baulast zu tragen hat, werden gegenwärtig Verhandlungen gepflogen ${ }^{65}$.

Auch über diese im Pfarrbericht von 1864 genannten Verhandlungen sind Akten im Pfarramt Eschental vorhanden. Unter anderem wurde Rechtsanwalt Scholl von Öhringen eingeschaltet, der erneut die Baupflicht klären sollte. Doch dieser konnte keine neuen Erkenntnisse zu Tage bringen, als schon in dem Rechtsstreit von 1821 erfolgt war, und kam zu folgendem Ergebnis: Ich weiß nun nicht, wie es gegenwärtig mit dem Bauwesen steht, ob es sofort vorgenommen werden müßte, oder ob es noch im Anstand bleiben kann, und ich muß es den Beteiligten anheimgeben, einen Beschluß zu fassen, ob gegen Eschenthal ein Antrag auf vorläufige Tragung der Kosten zusteht. Öhringen, den 23. November $1864^{66}$.

Obwohl die Kapelle nicht mehr benutzt werden durfte, wurde im Jahre 1871 vom Langenburger Glockengießer Johann Christian Friedrich König (†20. Juni $1886^{67}$ ) eine Glocke für Rüblingen gegossen ${ }^{68}$. Interessanterweise geschah dieser Glockenguss acht Jahre nachdem die Kapelle wegen Baufälligkeit geschlossen worden war. Diese Glocke war $170 \mathrm{~kg}$ schwer, hatte einen Durchmesser von $57 \mathrm{~cm}$ und war $50 \mathrm{~cm}$ hoch. Sie soll aus einer alten, zerbrochenen Glocke umgegossen worden sein, wahrscheinlich jener aus dem Jahre 1733. Folgende Inschrift in lateinischen Großbuchstaben lief in vier Reihen um die Glocke: Für die Gemeinde Rüblingen gegossen von C. König in Langenburg anno 1871. Unter Leitung des Pfarrers Scheuermann, Anwalt Stapf und Michael Metzger, welcher einen namhaften Beitrag spendete. Verkünde laut den Bund der Taufe. Ruf uns zur Kirch und Unterricht und töne, wenn in unserem Laufe der Pilgerstab am Grabe bricht ${ }^{69}$.

Wenige Zeit darauf wurde die Rüblinger Kapelle dann abgebrochen. In der Kreisbeschreibung von 1968 steht zum Abbruch zu lesen, dass dieser 1871/72 durchgeführt worden sei. An der Stelle der Kapelle stehe heute ein Eisengerüst mit den beiden Glocken ${ }^{70}$. Dieser Abbruchzeitpunkt wurde auch so in die Landesbeschreibung übernommen ${ }^{71}$. Tatsächlich beschloss der Stiftungsrat in Eschental aber noch 1873, die Kapelle um 2500 Gulden restaurieren zu lassen. Da der Bauzustand aber eine Renovierung nicht mehr zuließ, wurde in einem weiteren Beschluss für den Abbruch votiert ${ }^{72}$. Am 30. Januar 1874 entschied der

Evangelisches Pfarrarchiv Eschental.

Evangelisches Pfarramt Eschental.

Deutscher Glockenatlas (wie Anm. 50), S. 100.

Landeskirchliches Archiv Stuttgart A 29 Bd. 1483

Evangelisches Pfarrarchiv Eschental.

Der Landkreis Öhringen (wie Anm. 2), S. 161 und 165.

Das Land Baden-Württemberg (wie Anm. 2), S. 214.

Rauser (wie Anm. 2), S. 176. 
Eschentaler Stiftungsrat, dass der Abbruch von der Gemeinde Rüblingen durchzuführen sei, die dafür die Abbruchmaterialien erhalten sollte ${ }^{73}$. Aus der Formulierung kann man schließen, dass der Abbruch noch nicht vollzogen war. Noch am 5. Februar 1874 war die Abbruchgenehmigung nicht eingetroffen ${ }^{74}$. Aber noch im Frühjahr 1874 erfolgte der Abbruch der Kapelle, wie ein Verzeichniß der Holzversteigerung über Einnahme von dem Abbruchmaterial vom alten Kirchlein in Rüblingen berichtet ${ }^{75}$. Darin wird auch der Verkauf von Steinen und Ziegeln erwähnt.

\section{Der geplante Wiederaufbau und die Entstehung des heutigen Glockenturms}

Lange Zeit setzte man sich für einen Wiederaufbau der Kapelle oder den Bau eines Betsaales ein. Akten und Baupläne darüber haben sich im Gemeindearchiv Kupferzell erhalten ${ }^{76}$. Ein Kirchenbaukomitee, bestehend aus neun Personen, tagte immer wieder von 1885 bis 1916 . Aus dem Jahre 1886 existieren Baupläne über den Neubau einer Kapelle.
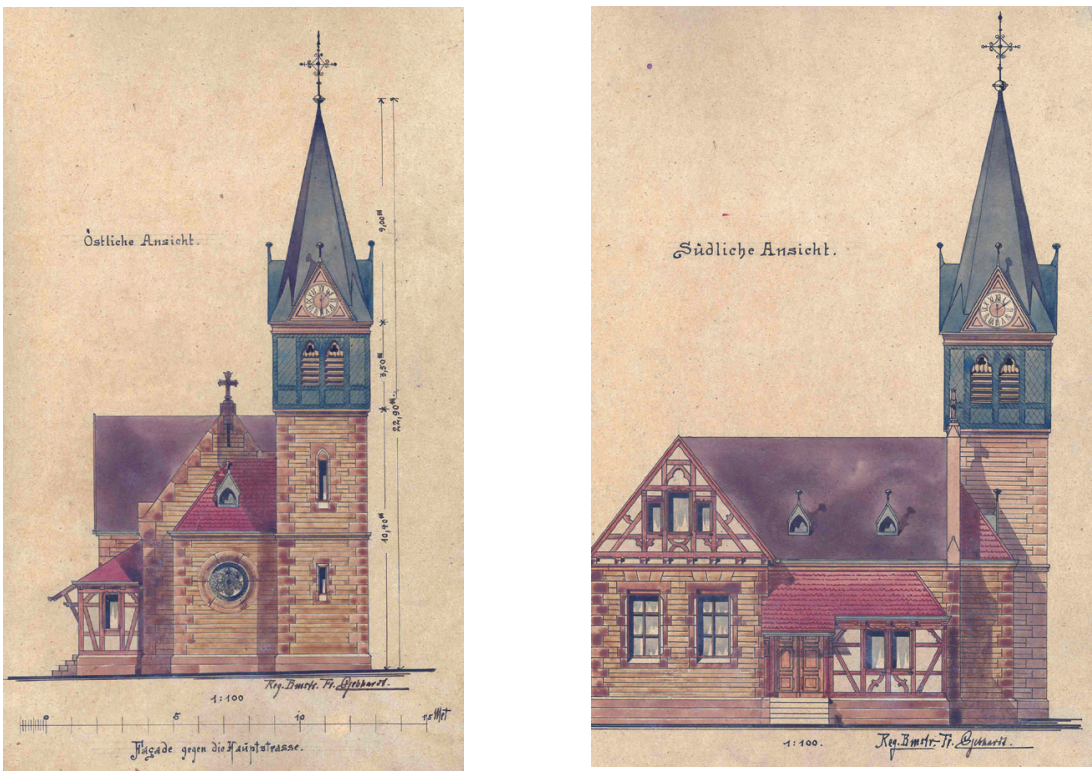

Abb. 2 und 3: Pläne über einen Kapellenneubau in Rüblingen von 1886

(Originale im Gemeindearchiv Kupferzell, Bestand Feßbach, A 337)

73 Evangelisches Pfarrarchiv Eschental.

74 Ebd.

75 Gemeindearchiv Kupferzell, Bestand Feßbach, A 336.

76 Ebd., A 337. 
Und 1890 wurden Pläne für einen Betsaal gefertigt. Ein geplanter Neubau wurde aber nie mehr verwirklicht.
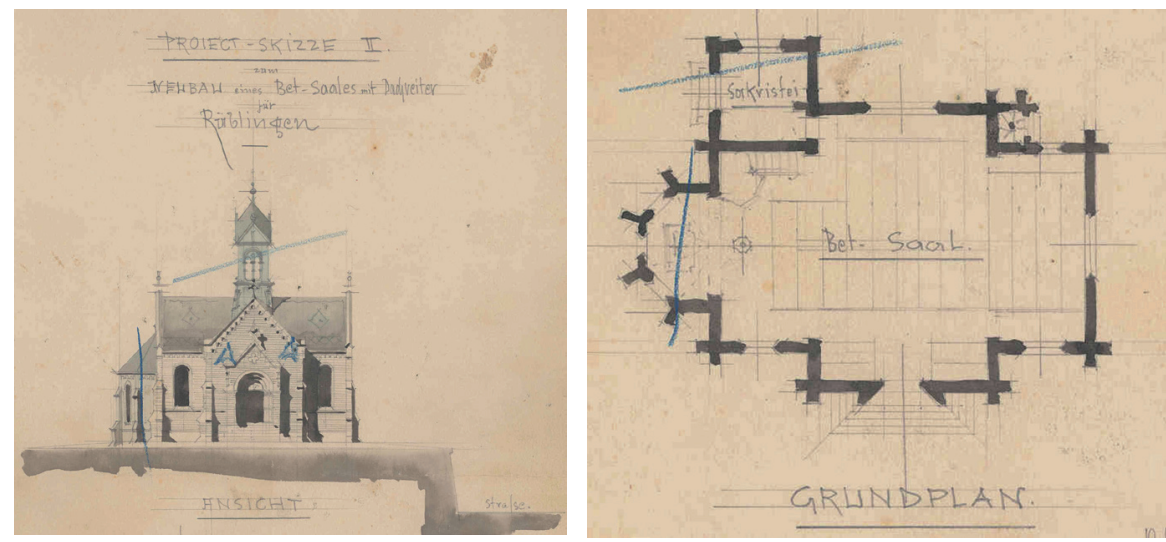

Abb. 4: Pläne über den Neubau eines Betsaales mit Dachreiter für Rüblingen von 1890 (Originale im Gemeindearchiv Kupferzell, Bestand Feßbach, A 337)

Beim Abbruch der alten St. Georgs-Kapelle in Rüblingen im Frühjahr 1874 hatte sich die Teilgemeinde Rüblingen die Glocken zurückbehalten. Da der Eschentaler Stiftungsrat den alten Kirchplatz als sein Eigentum betrachtete, suchte sich die Gemeinde einen anderen Standort, um die Glocken zu verwahren. Sie fand ihn beim Haus des Gemeinderats Johann Leonhard Metzger von Haus Nr. 1 (heute Waldstraße 36). Hier wurde ein Glockenschuppen von Fachwerk mit Pultdach errichtet, in dem nun die Stiftungspflege Rüblingen den Glockenstuhl von Eichenholz, eine Glocke mit $65 \mathrm{~cm} 240 \mathrm{~kg}$, eine Glocke mit $55 \mathrm{~cm} 240 \mathrm{~kg}$ aufstellte $^{77}$. Hier wäre anzumerken, dass die Gewichtsangaben mit Sicherheit falsch sind. Im Feuerversicherungsbuch von 1907 werden die Gewichte der Glocken mit $300 \mathrm{~kg}$ und $100 \mathrm{~kg}^{78}$, im Bericht des Evangelischen Konsistoriums Stuttgart anlässlich der Glockenabnahme 1917 mit $170 \mathrm{~kg}$ und $100 \mathrm{~kg}$ angegeben. Diese Abweichungen lassen sich nur damit erklären, dass das Gewicht immer nur geschätzt worden war. 
Erst mit der Ausscheidung des Kirchenvermögens gab der Eschentaler Stiftungsrat das Grundstück der abgebrochenen Kapelle frei. Im Stiftungsratsprotokoll vom 15. Mai 1891 heißt, dass der Kirchplatz zu Rüblingen [. . . zuerst als Eigentum der hiesigen Stiftungspflege betrachtet worden sei, nachdem man denselben aber im Güterbuch als Eigentum der politischen Gemeinde Rüblingen eingetragen habe, erhebe die Kirchengemeinde Eschental keinen Eigentumsanspruch mehr an diesen Platz ${ }^{79}$.

Doch zunächst hingen die Glocken noch in dem Glockenschuppen in der Waldstraße. Im Dezember 1894 machte der Öhringer Oberamtsbaumeister einen Kostenvoranschlag über die Reparatur der baufälligen Glockenremise und des Glockenstuhls in Rüblingen ${ }^{80}$, über den es 1907 im Feuerversicherungsbuch hieß: Am Ortsweg freistehend der Glockenschuppen von offenen und mit Latten vertäfeltem Fachwerk mit Pultdach von Dachplatten im oberen Weiler ${ }^{81}$. In der Rüblinger Überlieferung heißt es, als die letzte Mesnerin im Jahre 1914 verstarb, soll am Tag ihrer Beerdigung der Bretterverschlag zusammengefallen sein ${ }^{82}$. Wie viel Wahrheit in dieser Überlieferung steckt, lässt sich wohl nicht mehr feststellen.

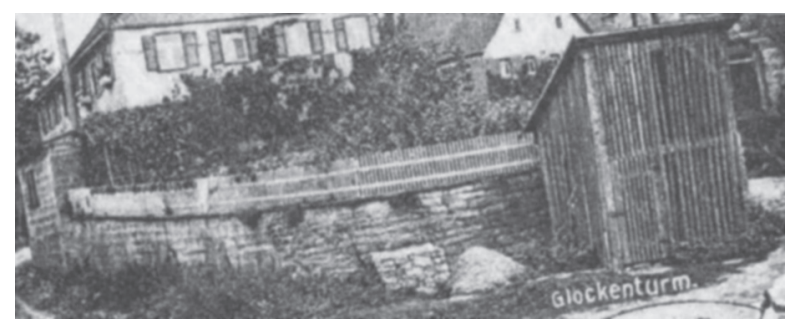

Abb. 5: Der Glockenschuppen auf einer Postkartenansicht um 1900

Widersprüchlich sind die Angaben zur Ablieferung der Glocken im Ersten Weltkrieg. Im Eschentaler Pfarrbericht von 1920 heißt es, dass beide Glocken abgeliefert wurden ${ }^{83}$. Dagegen berichtet der Feßbacher Schultheiß Stegmüller, dass eine von zwei Rüblinger Glocken Anfang 1917 habe abgeliefert werden müssen $^{84}$. Im Jahre 1920 wollte der Eschentaler Pfarrer Gmelin dafür sorgen, dass die Glocke nicht weiter am Balkengerüst und freiem Feld aufgehängt sei. Er konnte aber nichts erreichen, weil die Glocke Eigentum der bürgerlichen Gemeinde war $^{85}$.

79 Evangelisches Pfarrarchiv Eschental.

80 Gemeindearchiv Kupferzell, Bestand Feßbach, A 338.

81 Gemeindearchiv Kupferzell, Bestand Feßbach, B 158.

82 Rauser (wie Anm. 2), S. 176.

83 Evangelisches Pfarrarchiv Eschental.

84 Gemeindearchiv Kupferzell, Bestand Feßbach, A 338.

85 Evangelisches Pfarrarchiv Eschental. 
Schließlich rangen sich die Rüblinger dazu durch, einen Glockenturm errichten zu lassen. In dem schon erwähnten Bericht von Schultheiß Stegmüller heißt es: Nach Ende des Krieges hat Rüblingen eine zweite Glocke nicht mehr beschafft, sie begnügen sich mit einer Glocke. Für diese Glocke haben die Rüblinger Einwohner an Stelle eines erbärmlichen Glockenschuppens im Jahr 1921 den 25. Juli einen neuen eißernen Glockenstuhl mit dem Einbau einer neuen Uhr mitten im Ort an der Stelle, auf welcher die abgebrochene Kirche stand, erstellt. [Für] diese Kosten haben die Rüblinger einen Betrag von 9400 Mark dem Lieferanten Turmuhrfabrikanten Hörz in Ulm aus freiwilligen Beiträgen gezahlt. Auch dieses Unternehmen möge den Rüblingern ewig zum Frieden dienen ${ }^{86}$. Doch auch hier gibt es ein widersprüchliches Datum. Denn ein Nachtrag im Feuerversicherungsbuch von 1907 erwähnt, dass der Abbruch des Glockenschuppens und Bau des Glockenturms im Jahre 1925 gewesen sein soll ${ }^{87} .1925$ wurde folgendes zur Brandversicherung eingeschätzt: Am Ortsweg freistehender Glockenturm mit Uhrwerk auf Eisengestell ohne Dach. Dazu gehörten ein eiserner Glockenstuhl, eine Glocke mit $65 \mathrm{~cm}$ Durchmesser und $100 \mathrm{~kg}$ Gewicht sowie eine weitere Glocke für das Schlagwerk mit einem Durchmesser von $30 \mathrm{~cm}$ und einem Gewicht von $30 \mathrm{~kg}$. Demnach scheint anlässlich des Glockenturmbaus wieder eine zweite Glocke angeschafft worden zu sein. Außerdem wird im Feuerversicherungsbuch ein Uhrwerk von Herz in Ulm genannt. Da nach den Unterlagen der heute noch existierenden Firma Philipp Hörz die Uhr vom Typ T 165c im Glockenturm Rüblingen mit der Fertigungsnummer 2787 schon 1920 geliefert worden sein soll ${ }^{88}$, so dürfte das Jahr 1921 am ehesten für die Erstellung des Rüblinger Glockenturms zutreffen. Die Firma Hörz aus Ulm hat den Glocken- und Uhrenturm in Rüblingen auch im Jahre 1982 von Grund auf saniert ${ }^{89}$.

\section{Mündliche und volkstümliche Überlieferungen um die St. Georgs-Kapelle}

Von der Kapelle in Rüblingen heißt es schon 1681, dass sie einmal die Hauptkirche war und Eschental zu Rüblingen kirchlich zugeordnet gewesen sein soll ${ }^{90}$. Schon die 1454 belegte Bezeichnung „Kapelle“ spricht dafür, dass Rüblingen nicht die Mutterkirche von Eschental gewesen sein kann. Eine Kapelle war unselbständig und hatte keinen eigenen Pfarrer ${ }^{91}$. Nur die Pfarrkirche war Sitz eines Pfarrers, in unserem Falle war dies die Pfarrkirche von Eschental. Wenn 1486

86 Gemeindearchiv Kupferzell, Bestand Feßbach, A 338.

87 Gemeindearchiv Kupferzell, Bestand Feßbach, B 158.

88 Laut Auskunft von Erich Müller von der Philipp Hörz GmbH in Biberach.

89 Rauser (wie Anm. 2), S. 213.

90 Vgl. Anhang 1 und 2.

91 Gerhard Taddey: Die Kirche im Dorf. Eine historische Einführung. In: Peter Schiffer (Hg.): Die Kirche im Dorf. Sigmaringen 1998 (= Forschungen aus Württembergisch Franken 45), S. 8. 
der Eschentaler Pfarrer Johannes Zawer seine Einwilligung zum Tausch von Gütern des Rüblinger Heiligen gab, spricht auch dies gegen die mündliche Überlieferung, dass Rüblingen einmal die Mutterkirche der Eschentaler Kirche war. Zudem gibt es keinen einzigen Hinweis darauf, dass es in Rüblingen einmal einen eigenständigen Pfarrer gab. Die Predigten wurden - zumindest seit 1543, also schon vor Einführung der Reformation - nach Ausweis der ältesten Heiligenrechnung immer vom Eschentaler Pfarrer gehalten ${ }^{92}$. Nach Ausweis der Akten wurden in Rüblingen zwei Predigten gehalten. Dies findet sich auch in einem Verzeichnis der Güter und Gefälle der Pfarrei Eschental aus dem Jahr 1588. Dort wird erwähnt, dass der Eschentaler Pfarrer $1 \mathrm{fl}$ von den Heylgenpfleger Rüeblingen erhält, darumb weil Pfarrer auf Georgy und dan auch Rüeblinger Kirchwei mit Predig" versieht ${ }^{93}$. Außerdem erhielt der Eschentaler Schulmeister 40 x wegen des Gesangs ${ }^{94}$.

Als 1796 mit den Vorarbeiten für eine geplante topografische Beschreibung in den Hohenloher Landen begonnen wurde, berichtete der Waldenburger Hofrat Johann Christian Friedrich Herwig (1765 bis 1832): Rüblingen, hat ein Kirchlein, in welchem vor alten Zeiten Frühmessen gelesen wurden und dahin ein Walfarth zum Ritter St. Jörgen gewesen seyn soll. Dessen Mähren davon kann das Lagerbuch in Kupferzell bestimmen, bey hiesigem Pfarramt findet sich hierüber kein Actenstück noch alte Urkunde vor ${ }^{95}$. Leider ist das Kupferzeller Lagerbuch nicht erhalten geblieben, so dass diese Herwig'sche Aussage nicht überprüft werden $\mathrm{kann}^{96}$. Das Gerücht von der angeblichen Mutterkirche zu Eschental hielt sich hartnäckig. Als - wie oben gezeigt ${ }^{97}-1819$ eine Diskussion über die Unterhaltungskosten an der Rüblinger Kapelle aufkam, wurde in den Streit- und Prozessakten immer wieder auf diesen Umstand hingewiesen.

Angesichts der Tatsache, dass in dem Rechtsstreit viele - auch legendäre - ÄuBerungen gemacht wurden und dass keiner mehr wusste, von wem und wann die Kapelle erbaut wurde, verwundert es nicht, wenn Pfarrer Hörlin im Eschentaler Pfarrbericht von 1864 schrieb, dass die Kirche in Rüblingen der Sage nach die älteste Kapelle in Franken sei ${ }^{98}$. Zwei Jahre später war es dann schon keine Sage mehr, sondern Tatsache, denn im Pfarrbericht von 1866 heißt es: Die Kirche in Rüblingen, die älteste in Franken. Im Pfarrbericht von 1868 schrieb Pfarrer Hörlin dann schon wieder vorsichtiger: Die Kirche in Rüblingen, wohl die älteste in Franken. Pfarrer Scheuermann schrieb im Pfarrbericht von 1870: Die nach Han-

96 In HZA Neuenstein Wa 80 Bü 566 heißt es 1798, dass das Kupferzeller Lagerbuch schon seit ohnerinnerlichen Zeiten vermi $\beta$ t wurde.

97 Vgl. oben Kap. „Verfall der Kapelle [...]“.

98 Evangelisches Pfarrarchiv Eschental. 
selmann älteste Kirche im Filial Rüblingen und berief sich wohl auf den hohenlohischen Archivar Christian Ernst Hanselmann († 1775).

Ein anderes Kapitel sind die Rüblinger Streiche, im Dialekt die Rüwlinger Straach. In diesen Überlieferungen gibt es eine 1909 veröffentlichte Erzählung „Warum die Rüblinger keine Kirche haben“"99. Darin heißt es: „Die Kirche trug die Jahreszahl 1452 und war die Mutterkirche der umliegenden Weiler und Höfe." Hier erscheint also auch wieder das Motiv der Mutterkirche. Das angebliche Baujahr 1452 weist immerhin in ein von schriftlichen Quellen belegtes Jahrhundert. Manchmal enthalten diese „Streiche“ also auch einen wahren historischen Kern. In der gleichen Erzählung kommt nämlich auch der Kirchturm vor, der sich vom Langhaus hinweg neigte ${ }^{100}$. Was im Moderationsprotokoll von 1682 aber seine Ursache im weichen Boden hatte, ist in der Erzählung natürlich etwas schalkhafter dargestellt. In der Erzählung heißt es: „Die Einwohner zeichneten sich durch besonderen Verstand und Frömmigkeit aus, so dass der Segen von oben nicht ausblieb und sogar auf dem Dach der Kirche Gras wuchs. Die Rüblinger wollten die edle Gottesgabe nicht zugrund gehen lassen und machten's nun so: Sie zogen den Gemeindehummel an einem Seil hinauf, damit er das Gras abweide. ,Dös Groos mooch er gäre, er schleckt scho dernoch!' jubelten sie in ihrer kindlichen Freude, als das Tier beim Ersticken die Zunge heraushängte. Durch das Hinaufziehen des Hummels kamen die Rüblinger aber in großen Schaden. Turm und Kirchdach trennten sich voneinander, so dass das ganze Gebäude im vorigen Jahrhundert abgebrochen werden musste. Rüblingen ist seitdem nach Eschental eingepfarrt." Wie gesagt, das Ergebnis im Streich war das gleiche wie in der 1682 überlieferten Baubeschreibung: „Turm und Kirchendach trennten sich voneinander.“

\section{Kirchliche Handlungen und Beerdigungen in Rüblingen}

Nur die Hauptkirche mit ihrem Pfarrer war mit umfassenden Rechten ausgestattet und nur dort durften in der Regel Taufen und Begräbnisse durchgeführt werden. Eine Kapelle war unselbständig und hatte somit nach kirchlichem Recht auch kein Begräbnisrecht ${ }^{101}$. Im Falle von Rüblingen war die Pfarr- oder Hauptkirche in Eschental. Wie erwähnt, hatte 1543, schon vor der Reformation der Eschentaler Pfarrer zweimal im Jahre jeweils drei Messen in Rüblingen zu halten $^{102}$. Nach der Reformation hatte der Eschentaler Pfarrer jährlich zweimal zu

99 Württembergische Volksbücher - Lustige Geschichten aus Schwaben. Hg. vom Württembergischen Lehrer-Unterstützungsverein. 4. Bd. Stuttgart 1909, S. 64.

100 Vgl. Anhang 2.

101 Taddey (wie Anm. 91), S. 8.

102 HZA Neuenstein Wa 265 Bü 1950: 1 Gulden dem Pfarer von Eschentall von 6 Mess zu Ryblingen zu halten nemlich an Sanct Jörgen Tag 3 Mess und der rechten Kirben zu Ryblingen 3 Mess. 
predigen, einmal zu Georgi (23. April), einmal zur Kirchweih nach Michaelis (29. September) $)^{103}$.

Nur im Winter und bei schlechtem Wetter wurden Taufen und Trauungen in Rüblingen vorgenommen. Darüber berichtet schon das Lagerbuch von $1681^{104}$. Wurde die Hochzeit in Rüblingen abgehalten, so wurde peinlich genau darauf geachtet, dass der Hochzeiter seine Hochzeit dem Herkommen gemäs zu Rüblingen ausrichtet, oder bey vor waltens erheblichen Umständen sich dermaßem mit dem Würth zu Rüblingen abfinde, daß dieser schad- und klaglos gehalten und deßen Würthschaft hierunter kein Abbruch oder Nachtheil zu gezogen wer$d e^{105}$.

Zwar war um die Kirche ein Kirchplatz mit einer Kirchhofmauer, aber damit war kein Begräbnisplatz verbunden. Im Eschentaler Totenregister werden vor 1835 keine Bestattungen in Rüblingen erwähnt; diese wurden in Eschental vorgenommen. Erst als sich durch eine Seuche die Bestattungen vermehrten, wurde in Rüblingen ein Friedhof nordwestlich des Orts angelegt ${ }^{106}$. Tatsächlich finden sich im Eschentaler Totenregister von September 1834 bis Februar 1835 insgesamt 18 Sterbefälle aus Rüblingen, die alle am hitzigen Gallenfieber, Nervenfieber oder Schleimfieber starben ${ }^{107}$. Nur ein Todesfall fällt in dieser Zeit aus dem Rahmen, wo der Tod anlässlich Brandweinrausch eintrat. Wollte sich hier jemand durch Alkohol vor der Seuche immun machen?

Die Tradition berichtet aufgrund der vielen Todesfälle weiter: Ein Rüblinger edelgesinnter Bürger namens Frank faßte deshalb den Entschlu $\beta$, in Rüblingen einen Kirchhof auf seine Kosten anzulegen, was er dann auch zur Ausführung brachte. Nun aber wollte es die Vorsehung, daß gerade der Stifter der erste sein sollte, der darin begraben wurde. Der Pfarrer von Eschental erhielt nach der Anlage des Friedhofs $183510 \mathrm{fl}$ aus der Gemeindekasse als Reisekostenersatz bei Beerdigungen ${ }^{108}$. Bei dem Kirchhofstifter handelte es sich um Johann Michael Frank, der tatsächlich als erste Bestattete im neuen Rüblinger Friedhof bestattet wurde ${ }^{109}$.

103 HZA Neuenstein Wa 260 Bd 84: jährlich zwey Predigten; alß die eine am Tag Georgy |: welchen die Rüblinger alß Ihren Kirchenpatronen annoch feyerlich begehen, undt sich am selbigen das Heylig Abendtmal, wer deßen benöthiget, reichen laßen :| undt die andern an Ihrer Kirchwey, nach Michaelis.

104 Vgl. Anhang 1.

105 HZA Neuenstein La 35 Bü 3329.

106 Rauser (wie Anm. 2), S. 208: Anfangs der Dreißiger Jahre wütete in genannter Kirchengemeinde eine heftige Seuche, und die Verstorbenen (der Würgengel forderte über 30 Opfer) mußten damals noch in den Kirchhof nach Eschental getragen werden. Die Entfernung beider Gemeinden ist aber ziemlich beträchtlich und der Weg namentlich sehr uneben, wodurch der Transport der Leichen sehr erschwert wurde.

107 Evangelisches Pfarrarchiv Eschental.

108 Ebd..

109 Ebd.: In Franks Sterbeeintrag im Eschentaler Kirchenbuch heißt es: Das war das erste Begräbnis im neuen Kirchhof daselbst. 
Aus den Akten wird deutlich, dass die Bürger von Rüblingen sich 1835 die Unzulänglichkeiten im Eschentaler Friedhof zu Nutze gemacht hatten, um einen eigenen Friedhof anzulegen. In einem Schreiben vom 16. Juli 1835 an das Oberamt in Öhringen wird darauf hingewiesen, dass man den Rüblinger Friedhof u. a. auch deshalb angelegt habe, weil im Eschentaler Friedhof die Beerdigten [...], besonders bey sich vermehrenden Sterbesläufen, wie wir im verflossen und heurigen Jahr hatten, nicht gehörig verwesen könnten ${ }^{110}$.

\section{Das Patrozinium des heiligen Georg}

Der heilige Georg war der Schutzherr oder Schutzheilige der Kapelle, ihm war die Kapelle geweiht. Er hatte über den Besitz und das Vermögen der Kapelle zu wachen, welches sie durch Schenkungen, Käufe oder Tauschverträge erhalten hatte. Mit dem heiligen Georg ist Georg von Kappadokien gemeint, welcher um 303 n. Chr. den Märtyrertod starb. Georg ist einer der vierzehn Nothelfer und galt als Schirmherr der Kreuzfahrer und des Deutschen Ordens ${ }^{111}$. Georgs Lebensbeschreibung scheint sich nur aus Legenden zusammenzusetzen, die allerdings schon früh bekannt wurden. Danach stammte er aus Kappadokien, kämpfte als römischer Legionär an vielen Enden des Imperiums Romanum, wurde Christ und erlitt in den Christenverfolgungen unter Kaiser Diokletian ein Martyrium besonders entsetzlicher Art. Ab dem 11. Jahrhundert lässt sich das Motiv des Drachenkampfes nachweisen, das den Heiligen bis heute begleitet. Durch die Kreuzzüge lebte die Verehrung des heiligen Georg neu auf.

Wie die Rüblinger Kapelle zu dem heiligen Georg als Schutzpatron kam, ist nicht bekannt. Die Wahl des Schutzpatrons übernahm in der Regel der Kirchengründer. Wer der Kirchengründer in Rüblingen war, war schon im Lagerbuch von 1681 unbekannt. Dort wird auch eine jährliche Feier am Georgstag erwähnt ${ }^{112}$. Über einige Regeln des Georgstages in Rüblingen wird noch 1770 berichtet ${ }^{113}$. Bei diesem Kirchenfest handelte es sich aber nicht - wie man vermuten würde - um ein Kirchweihfest. Eine solche Kirchweihe fand in Rüblingen nach Michaelis (29. September) statt.

110 StAL F 192 II, Bü 970.

111 Im Folgenden nach Erhard Gorys: Lexikon der Heiligen. München 1997, S. 113.

112 Vgl. Anhang 1.

113 Gemeindearchiv Kupferzell, Bestand Feßbach, A 366: Das an heut zu endgesechsten Dato ist beym Abrechnen in der Gemeind fest geschlosen worden, das am Samstag Morgen die Besserungs u[nd] Erdenfuhren sollen erlaubt sein. Nachmittags aber bey 30 Kreuzer Straff verbotten sein, das Unterackern soll erlaubt sein, weilen aber wie gewöhnlich der Georgy Tag bißher nur halb gefeyert worden, so soll er von einem jeden Gemeindsmann auch Kinder u[nd] Gesind der Tag gantz gefeyret werden wie andere Feyertäge auch. Rübl[ingen], den 25ten Febr[uar] 1777. 


\section{Anhang 1: Die kirchlichen Verhältnisse in Rüblingen $1681^{114}$}

In dem Dorff Rüblingen hat es ein altes Kirchlein, welches biß dahero von deß Heyligen zu Eschenthal Zinßgeltern im Bau erhalten worden, unbewust aber, wer es anfangs erbauet, dann es vor alters, wie aus alten Gültbüchern zu ersehen, mit aigene Heyligem, Gültten undt Einkunften versehen gewesen, undt solle, nach Aussag der Alten, diß Kirchlein hiebevorn der Pfarr Eschenthal, dahin es jetzo ein Filial, Mutterkirch gewesen seyn. Ein Pfarrer zu Eschenthal muß |: wie herkomblich :| in diesem Kirchlein jährlich zwey Predigten, al $\beta$ die eine am Tag Georgij |: welchen die Rüblinger, alß ihrem Kirchen Patronen annoch feyerlich begehen, undt sich am selbigen das Heilig Abendmahl, wer deßen benöthiget, reichen lassen :| undt die andere an ihrer Kirchwey nach Michaelis ablegen, dafür hat er auß deß Heyligen Zinnßgelttern, jährlich einen Gulden undt der Schuelmeister wegen deß Gesangs viertzig Kreutzer zu gewartten. Werden auch underweilen verheiratete Personen in diesem Kirchlein, wann sonderheitlich das Hochzeitsmahl in selbigem Flecken gehalten wird, copuliert, undt öfters die Kinder darin getauft, bevorab zu Winterszeit undt wann böses Wetter. Sonsten aber seyndt die Innwohner solchen Orts schon von alters hero nacher Eschenthal, wo selbst meiner gräflichen Herrschaft das Ius Episcopale gepfarret, allda sie auch ihre Begräbnis haben undt alle Kirchenactus exercieren. Solchem nach dann die geistliche Jurisdiction solchen Orts, wo sich jechtwas hierinnen zu entscheiden bei den Inwohnern zuträget, vor das Consistorium nacher Waldenburg gehörig.

\section{Anhang 2: Beschreibung der Rüblinger Kapelle $1682^{115}$}

Ein Moderationsprotocoll, welches 1682 anlässlich einer hohenlohischen Landesteilung angelegt wurde, bringt folgende kurze Beschreibung der Rüblinger Kapelle: Daß Kirchengebäw zu Rüblingen sampt der Ringmauer und Thurm befindet sich alles in feinen, gueten weßentlichen Baw nur dieses ist darbey in Consideration zu nehmen, daß so viel man erkennt, sich der Thurm vom Langhauß hinweg begeben will, angesehen er zwar noch nach uffrecht stehet, doch der Grundt, weilen er uff einem Berglein, nachgeben undt senckhen müße, undt waß biß hero zu bawen uffgewendet worden, ist alles von dem Eschenthaler Heyligen, davon selbige Kirch auch erhalten wirdt, gefallen, die Glockhen aber erhaltes die Gemeind, auff dero aigene Costen, und wo der Heyligen nicht erkläcklich, hat gnädige Herrschaft, weilen sie einige Heyligengefälle an sich gezogen, zu mahlen die Oberinspection undt eine Nutrix solchen Gotteshaußes, die Uncosten beyzutragen, undt zu erhalten, [...] Sonsten aber seind die Inn- 
wohner solchen Orts schon von alters hero nacher Eschenthal, wo selbst meiner gräflichen Herrschaft das Ius Episcopale gepfarret

\section{Anhang 3: Zum Glockenguss 1733}

Anhang 3.1: Abrechnung des Glockengusses ${ }^{116}$

Die neue Glock zu Rüblingen hat gewogen: 2691/2 Pfund laut Waagzettels. Hiervon werden für 256 $1 / 2$ Pfund umzugießen nach dem Accord á 8 Kreuzer vom Pfund bezahlt: 40 Gulden 8 Kreuzer. Für 13 Pfund neu darzu gegebenen Zeig á 32 Kreuzer: 6 Gulden 56 Kreuzer. Ferner pro 1 Pfund Wachs 30 Kreuzer. Zehrung 34 Kreuzer. Dem Schreiner vor ein Joch und Gestell uffem Rüblinger Kirchturm zu machen 1 Gulden. Dann ist für Holz und andere Sachen ausgelegt worden 4 Gulden 37 Kreuzer. Summa: 54 Gulden 15 Kreuzer. Welche fünfzig vier Gulden fünfzehn Kreuzer aus dem Eschenthaler Heiligen zu bezahlen und hiermit in Ausgabe zu bringen sind. Eschenthal, den 27. Novembris 1732.

\section{Anhang 3.2: Verhandlungen mit dem Glockengießer Lösch ${ }^{117}$}

Der Glockengießer Johann Lösch in Morspach verlangt von zersprungenen Glocken in Rüblingen, welche bey 2 Centner wägen solle, vom Pfundt, wenn ers allein gießen solle, 10 Kreuzer, 2 Claffter Holz und 1 Pfund Wachs. Hergegen wann ers mit andern bereits unter Hande habenden in einen Guß bringen dörffe 8 Kreuzer, wobey er den Abgang allein leiden und das völlige Gewicht wieder geben, auch Jahr und Tag die Gewehrschaft, daß die Glocke guth und tüchtig, leisten will. Sollte aber ein Zusaz verlangt werden, so will er das Pfundt guten Zeug durchaus nicht anders geben als vor 32 Kreuzer, welches hiermit hoher gnädigster Herrschaft unterthänigst berichten und zugleich die gnädigste Resolution hirrüber erwarten wolle. Eschenthal, den 4. Oktobris 1733.

\section{Anhang 3.3: Vertrag mit dem Glockengießer Lösch ${ }^{118}$}

Mit Löschen, Glockengießer in Morspach ist wegen der Rüblinger zersprungenen Glocken so beyläuffig 2 Centner wägen soll, folgender Accord geschlossen worden: Erstens daß diese Glocke mit andern bereits in Händen habend Glocken, die auch von güten Zeüg zue seyn versichert worden, umgegossen, und also verfertigt werden soll, daß aller Abgang von ihme Glockengießer allein gelitten, und das vorige Gewicht von ihme wird geschafft, wenig oder nicht viel Zusaz über ihr jeziges Gewicht, um die Kosten zu erspahrn von ihr gege-

116 Gemeindearchiv Kupferzell, Bestand Eschental, R 289.

117 Ebd., Rechnungsbeleg Nr. 7.

118 Ebd. 
ben, und im solche guten Standt geliefert werden solle, daß er Jahr und Tag dahier Gewehrschafft leisten kann. Zweitens wogegen ihme versprochen worden, die Glocke nach Morspach zu lieffern, und daselbst in Beysein des Schultheißen von Rüblingen Johann Georg Strecker wägen zu lassen, auch 8 Kreuzer von dem Pfundt umzugießen zu bezahlen, ingleich 2 Claffter Holz zu lieffern, und 1 Pfund Wachs. Drittens solte aber etwas weniger Zusaz darzu kommen, solle ihme das Pfund guter Zeug á 32 Kreuzer bezahlt werden, welches wir einander hiermit versichern wollen. Rüblingen, den 8. Oktobris 1733.

\section{Anhang 3.4: Waagzettel zum Glockenguss ${ }^{119}$}

Cüntzelsau den IVten October 1733. Lassen die Herrn von Rüblingen in allhisiger gemeiner Waag eine allte Glocken wegen. Wigt im Globen 259 Pfund geht ab Globen Gewicht 2 1/2 Pfund verbleibt allso netto 256 1/2 Pfund. Wird ein solches von un $\beta$ beden Waagmeistern bescheindt, als Johann Adam Model, Johannes Schmetzer. Nach der Fertigstellung der Glocke heißt es in einem weiteren Waagzettel: Cüntzelsau den 9ten November 1733. Wird in allhiesiger Waag eine Glocke welche gehörig nacher Rüblingen gewogen. Wigt 272 Pfund wird ein sollches von uns Waagmeistern atestiert. Joh. Adam Model und Johannes Schmetzer. Nota: Von den 272 Pfund wird wieder am Globen Gewicht 2 1/2 Pfund abzuziehen seyn, verbleiben also nicht mehr als 269 1/2 Pfund.

119 Ebd. 
